\title{
The response to patient deterioration in the UK National Health Service - a survey of acute hospital policies
}

Mr Simon Freathy*, RN, BSc, PgD,

Venous Thromboembolism Specialist Practitioner, Portsmouth Hospitals NHS Trust,

Queen Alexandra Hospital, Portsmouth PO6 3LY

UK

Professor Gary B Smith, FRCA, FRCP,

Centre of Postgraduate Medical Research \& Education (CoPMRE),

Faculty of Health and Social Sciences,

Bournemouth University,

Bournemouth BH1 3LT

UK

Professor Lisette Schoonhoven**, PhD,

Professor of Nursing,

Faculty of Health Sciences and National Institute for Health Research Collaboration for Leadership in Applied Health Research and Care (Wessex),

University of Southampton,

Southampton SO16 6YD

UK

Professor Greta Westwood ${ }^{* *}, \mathrm{PhD}, \mathrm{RN}$,

Deputy Director of Research \& Director of Clinical Academic Practice,

Portsmouth Hospitals NHS Trust \& University of Southampton,

Portsmouth Hospitals NHS Trust,

Queen Alexandra Hospital,

Portsmouth PO6 3LY

UK

Corresponding author:

Professor Gary B Smith, FRCA, FRCP

Centre of Postgraduate Medical Research \& Education (CoPMRE),

Faculty of Health and Social Sciences,

Bournemouth University,

Bournemouth $\mathrm{BH} 13 \mathrm{LT}$

UK

Tel: 01202962782

Email:gbsresearch@virginmedia.com

Word count $=2599$

Abstract word count $=250$

Number of references $=26$

Figures $=4$

Tables $=2$

Key words: Early warning scores; National Early Warning Score; Patient deterioration; Policies;

Rapid response systems.

*now, Senior Clinical Quality Officer, NHS South Eastern Hampshire CCG, Hampshire, UK

**now, Professor of Nursing Science, UMC Utrecht, Utrecht, The Netherlands

${ }^{* * *}$ now, Chief Operating Officer, Florence Nightingale Foundation, London, UK 


\section{ABSTRACT}

Background: The assessment of acute-illness severity in adult non-pregnant patients in the United Kingdom is based on early warning score (EWS) values that determine the urgency and nature of the response to patient deterioration. This study aimed to describe, and identify variations in, the expected clinical response outlined in 'deteriorating patient' policies/guidelines in acute NHS hospitals.

Methods: A copy of the local 'deteriorating patient' policy/guideline was requested from 152 hospitals. Each was analysed against pre-determined areas of interest, e.g., minimum expected vital sign observations frequency, expected response and expected staff response times.

Results: In the 55 responding hospitals (36.2\%), the documented structure and process of the response to deterioration varied considerably. All hospitals used a 12-hourly minimum vital signs measurement frequency. Thereafter, for a low-risk patient, the minimum frequency varied from ' $6-12$ hourly' to 'hourly'. Frequencies were higher for higher risk categories. Expected escalation responses were highly individualised between hospitals. Other than repeat observations, only nine (16.4\%) documents described specific clinical actions for ward staff to consider/perform whilst awaiting responding personnel. Maximum permitted response times for medium-risk and high-risk patients varied widely, even when based on the same EWS. Only $33 / 55$ documents $(60 \%)$ gave clear instructions regarding who to contact 'out of hours'.

Conclusions: The 'deteriorating patient' policies of the hospitals studied varied in their contents and often omitted precise instructions for staff. We recommend that individual hospitals review these documents, and that research and/or consensus are used to develop a national algorithm regarding the response to patient deterioration. 


\section{INTRODUCTION}

In 2012, the Royal College of Physicians of London (RCPL) recommended standardising the assessment of acute-illness severity for non-pregnant, adult patients using a single early warning score (EWS) - the National Early Warning Score (NEWS) ${ }^{1}$ [updated in 2017 to NEWS2 ${ }^{2}$ ]. NEWS allocates 0-3 points to measurements of each of six clinical variables - heart rate, respiratory rate, systolic $\mathrm{BP}$, temperature, conscious level evaluated using the AVPU scale, $\mathrm{S}_{\mathrm{p}} \mathrm{O}_{2}$ - based on their derangement from "normal". Two points are added for the use of supplemental oxygen when the vital signs are measured. ${ }^{1,2}$ The RCPL recommends that NEWS should be used to determine the urgency and nature of the response when a patient's clinical condition deteriorates, the frequency of subsequent clinical monitoring and the setting in which ongoing clinical care is delivered. ${ }^{1,2}$ In line with earlier suggestions by the United Kingdom's (UK) National Institute for Health and Care Excellence (NICE), ${ }^{3}$ the RCPL recommended a 12-hourly minimum frequency of observations. It also recommended that the frequency of subsequent patient observation and the response to deterioration

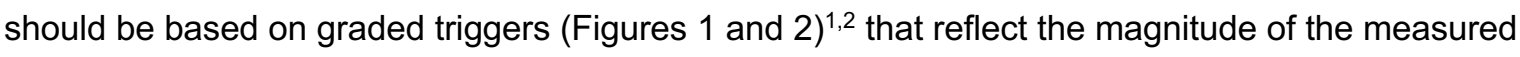
NEWS value. An individual parameter score of 3 (indicating 'low-medium risk') or an aggregate NEWS value of 5-6 (indicating 'medium-risk') should initiate an urgent clinical review. An aggregate NEWS value of 7 or more (indicating 'high-risk') should initiate an emergency clinical review. ${ }^{1,2}$ To reflect local differences in the composition of the response due to the size and case-mix of the hospital, the RCPL advised that the clinical response to acute illness should be agreed locally.

It could be expected that the specific local arrangements surrounding the response to deterioration in an acute hospital would be described within local hospital documents, such as "deteriorating patient" policies. These documents should describe the organization's expectation of when and how care should be escalated, the speed of the response, the grade and competencies of the responding staff, and the actions necessary to guarantee the expected response at all times. In addition, the documents could usefully describe the specific actions expected of ward first responders (usually ward nursing staff) in the time between calling for help and the arrival of a doctor, other appropriately trained practitioner or response team (e.g., Critical Care Outreach Team or Medical Emergency Team). Such actions should be based on the ABCDE principles as recommended on acute care 
courses $^{4,5}$ and could include repositioning the patient, airway suctioning, administering $\mathrm{O}_{2}$ therapy, IV fluids and consideration of prescribed medications.

The aim of our study was to examine acute National Health Service England (NHSE) hospitals' 'deteriorating patient' policies/guidelines to identify the specific local arrangements surrounding the response to deterioration and to describe the extent of variation across organisations of actions expected of staff following an EWS trigger.

\section{METHODS}

The Directors of Nursing/Chief Nurses of 152 acute NHSE Hospitals were contacted via an introductory email from the Director of Nursing, NHS Improvement (NHSI) in July 2017. This email explained the purpose and context of the study and requested a copy of the hospital's 'deteriorating patient' policies/guidelines for non-pregnant adult patients. A follow-up email was sent two months later to non-responding organisations.

The documents received were analysed by a member of the research team (SF) and results collated in a spreadsheet and compared against pre-determined areas of interest. These included:

- The EWS in use

- Whether the EWS included all six parameters recommended in NICE CG50

- Whether the EWS required the recording of supplemental oxygen therapy, as used in NEWS ${ }^{1,2}$

- The minimum expected frequency of vital sign observations for patients categorised by EWS as low- (i.e., EWS>0), medium- and high-risk.

- Whether a specific communication tool, e.g. Situation-Background-Assessment-Response $\left(\mathrm{SBAR}^{6}\right)$, was used during escalation

- The expected response to EWS escalation in normal 'office hours'

- The expected response to EWS escalation 'out of hours'

- The presence of any reference to extended nursing actions

- The expected staff response times for patients identified as at 'medium-risk' of deterioration

- The maximum time permitted for a 'medium-risk' response 
- The expected staff response times for patients identified as at 'high-risk' of deterioration

- The maximum time permitted for a 'high-risk' response

- The necessary actions following failure to implement the expected 'medium-risk' and 'highrisk' response (i.e., response failure)

- The presence of any reference to consideration to end-of-life care (EOLC) / do not attempt cardiopulmonary resuscitation (DNACPR) plans

In line with guidance from the NHS Research Authority, this evaluation of organisational policies and guidelines did not require ethical review by an NHS or Social Care Research Ethics Committee or management permission through the NHS R\&D office.

\section{RESULTS}

Policies/guidelines were received from 55/152 (36.2\%) acute NHSE hospitals. Overall, we observed considerable variation in the specific instructions regarding the structure and process of the response to deterioration, even when the organisations used the same EWS. There was variation in the type of EWS being used (Figure 3), with the majority (36/55 [65.5\%]) employing NEWS, often within electronic vital signs software such as Vitalpac ${ }^{\mathrm{TM}} .{ }^{7}$ All 55 hospitals collected the six minimum physiological observation parameters recommended in NICE CG50. ${ }^{3}$ However, 8 (14.5\%) of the EWS in use did not include the additional weighting score for patients requiring supplemental oxygen, as used in NEWS. ${ }^{1,2}$

Irrespective of the EWS in use, there was considerable variation in the minimum frequency of observations for the different risk categories (Table 1). All hospitals used a 12-hourly minimum frequency of observation, with many using more frequent minimum regimens. Thereafter, for a lowrisk patient with an EWS value $>0$, the minimum frequency for hospitals using NEWS ranged from 6 to 12-hourly to 4-hourly, whereas for hospitals using an EWS other than NEWS, the minimum frequency ranged from 12-hourly (5.3\%) to hourly (5.3\%). Some hospitals explicitly left nursing staff to decide upon the frequency for low-risk patients, whereas one policy document did not state a minimum frequency of observations. For higher risk categories, minimum observations frequencies were higher. Four (7.3\%) employed a 6-12 hourly schedule and another four (7.3\%) using 4-12 hourly schedule. 
Hospitals' use of specific communication tools for healthcare staff to describe a patient's condition also varied: 42 (76.4\%) used SBAR ${ }^{6}$ and two (3.6\%) used an SBAR-variant. One organisation permitted the use of two different systems concurrently, i.e., SBAR and Reason-Story-Vital SignsPlan $\left(\right.$ RSVP $\left.^{8}\right)$, whilst others used RSVP or REACT (Reason for calling-Extra clinical informationAssessment-Concerns-Treatment plan) as their sole system (1.8\%). From the deterioration policies provided, it could not be determined if eight organisations (14.5\%) used a specific communication tool.

The expected escalation response was highly individualised between hospitals, making it difficult to describe each in detail here. However, during 'normal office hours', the expected response to a lowrisk escalation was usually provided by a Registered Nurse, often the nurse in charge. For 'mediumrisk' escalations, the expected response was mostly by the ward medical staff caring for the patient, although this might also be in combination with Advanced Nursing Practitioners and/or Critical Care Outreach Team (CCOT) members. For high-risk escalations, the expected response was mostly by emergency staff not usually working on the ward (e.g., CCOT, Cardiac Arrest Team or Medical Emergency Team (MET) members), although 20 hospitals (36\%) appeared to rely exclusively on the ward medical staff caring for the patient as their first-line response. Only $33 / 55(60 \%)$ of the organisational policies and guidelines gave clear instructions regarding who to contact regarding the care of a deteriorating patient 'out of hours'. In order of frequency, staff were instructed to contact a dedicated a multidisciplinary team 'hospital at night' team, ${ }^{9}$ the Medical/Acute Response Team or the 'on-call' medical team.

Other than repeating observations, only nine (16.4\%) policies and guidelines provided clear instructions regarding specific clinical actions for nursing staff to consider/perform in the time between calling for help and the arrival of responding personnel. The remainder gave no instructions, apart from contacting the response personnel or team. When specific instructions were provided they included instituting simple measures, such as repositioning the patient, administering any 'as required' or emergency drugs including supplemental oxygen, and giving intravenous fluids, often under a 'patient group directive' (PGD). ${ }^{10}$ 
The maximum permitted response times for low-risk patients were rarely stated in policies. For medium-risk and high-risk patients, there was considerable variation between hospitals in the expected speed of response, even when they were based on the same EWS, i.e., NEWS (Table 2). Some hospitals used subjective terms such as 'urgent' and 'emergency', rather than using a specific time-frame such as 'within 15 minutes'. Where precise response times were given, they ranged from 'immediate' to 2 hours for medium-risk patients, and from 'immediate' to 1 hour for high-risk patients. Where hospitals used NEWS, maximum response times for medium-risk and high-risk patients were not stated in $4 / 36(11.1 \%)$ and $2 / 36(5.6 \%)$ of organisations, respectively. These proportions were higher for hospitals not using NEWS (Table 2). Guidance regarding the necessary corrective action for staff to take in the event of medium-risk and high-risk escalation failure were stated in only 32 $(58.2 \%)$ of policies and guidelines (Figure 4$)$.

Staff were asked to consider discussing EOLC and DNACPR with patient within the context of medium or high-risk EWS triggers in $26 / 55$ (47.3\%) documents.

\section{DISCUSSION}

In our survey of 55 acute NHSE hospitals' 'deteriorating patient' policies/guidelines we found considerable variation in the instructions provided to staff regarding minimum monitoring frequencies and maximum response times for responding staff. This variation was present, even when hospitals used the same EWS. We also observed that few hospitals provided clear instructions regarding simple clinical actions for bedside staff to undertake whilst awaiting the arrival of responding personnel. In addition, policies/guidelines often used ambiguous, and, therefore, potentially confusing, terminology.

This study has several strengths. To our knowledge it is the only research to examine expected responses to patient deterioration at hospital level across a health service. The researchers used an objective, systematic approach to interrogate the organisational policies and guidelines independently, and the analysis was not influenced by anyone external to the research team. The research was facilitated by an introductory email to the Directors of Nursing/Chief Nurses of 152 acute 
NHSE hospitals by the Director of Nursing, NHS Improvement (NHSI). A follow-up email was sent two months later to non-responding organisations. The documents received were all current at the time of request.

However, the study also has weaknesses. Despite the follow-up email the response rate was only $36.2 \%$, which may have introduced non-response and volunteer bias. Nevertheless, our findings would probably be unchanged (other than in magnitude) by information from non-responding hospitals, and the considerable variability in expected practice already identified in responding hospitals is clearly concerning. Even when hospitals did return 'deteriorating patient' documents, it is possible that we may have missed information relevant to our study because staff guidance is often distributed amongst several different documents within a single organisation. This raises questions about how hospitals disseminate essential service information to their clinical staff.

A recent baseline survey by NHSI of the use of NEWS in England had a higher return rate (83\%) than our survey, likely due to the fact that this was an officially mandated survey from a national body. ${ }^{11}$ It reported that $64.6 \%$ of responding organisations used NEWS without modification as their tool for directing the response to deterioration. This is almost identical to our finding of $65.5 \%$. However, the NHSI survey also identified that almost $100 \%$ of responding organisations claim to have a 'formal escalation process' for when a patient's clinical condition deteriorates. ${ }^{11}$ This statement conflicts with the results of our survey, where some 'response to deterioration' policies lacked important structural and process information.

Providing instructions to staff about the expected actions to be taken when patient deterioration is identified is a pre-requisite to ensuring the necessary, timely clinical interventions required to prevent adverse clinical outcomes 24-hours a day. ${ }^{12}$ When there is uncertainty about the required tasks, who should undertake them, the speed of the response or the corrective action to be taken when escalation does not occur, 'failure to rescue' is more likely. ${ }^{13-16}$ Therefore, the finding that many policies surveyed in the current research failed to include clear and precise instructions is concerning, as is the amount of variation observed between hospitals. 
However, in part, these findings may have resulted from the paucity of evidence that supports specific timings and actions. For instance, there is currently no evidence to support any specific minimum vital signs monitoring frequency, ${ }^{17}$ or how the observation frequency should increase when patients deteriorate. The optimal frequency is likely to depend not only upon the probability of adverse clinical events occurring, but also the monitoring modalities and staff available, as even with current recommended monitoring schedules ${ }^{1-3}$ and staffing levels, significant numbers of observations are missed. ${ }^{18,19}$ There is also little published data regarding the actions ward staff currently undertake in the period between calling for help and the arrival of responding personnel, or which of these interventions have impact. Likewise, the optimum speed of response to deterioration is unknown, although few acute clinical conditions improve when treatment is delayed. This makes it sensible to keep response times short, ${ }^{20}$ although again there is no evidence to support specific intervals.

One of the findings of our study that requires further investigation is the disparity of expected actions between different hospitals, even those using an identical EWS (e.g. NEWS). Whilst we accept that the RCPL advises that clinical responses to NEWS values should be agreed locally, it is important to note that all the organisations consulted in the current study are acute hospitals that are likely to have a generally similar patient case-mix and workforce. Consequently, the significance of a given NEWS value is likely to be similar in each of these sites and, therefore, in theory, should warrant the same type and speed of clinical response. Consequently, a greater degree of standardisation between hospitals could probably be achieved with resultant benefits, including consistency in clinical decisionmaking, opportunities to unify staff training, and improved quality of care and patient safety. ${ }^{21,22}$

Nevertheless, we are keen to emphasise that, although important, improving the response to patient deterioration is not simply a case of improving the detail in policy documents or standardising care between hospitals. ${ }^{23,24}$ Organisational policies and guidelines are merely vehicles for hospitals to define the delivery of clinical care, in line with recommendations from national bodies. Whilst significant effort and resources are utilised in developing clinical guidelines, much less effort is expended in their implementation and publication alone is unlikely to change clinician behaviour. Therefore, effort is required to ensure that staff are aware of the contents and implications of policy 
documents, and that these documents convey unambiguous statements about any compulsory clinical actions and their timings.

\section{RECOMMENDATIONS}

We recommend that individual hospitals urgently scrutinise their policies and standard operating procedures to ensure that their instructions to staff about how to respond to patient deterioration are clear and concise. Guidance should be relevant to the whole 24-hour period. Local audit should be used to monitor the compliance with policies regarding the response to deterioration and, where necessary, change implemented. ${ }^{25,26}$ In addition, we recommend that the NHS identifies a UK body to urgently develop a uniform unambiguous algorithm for responding to deteriorating patients in acute hospitals to complement NEWS and for national introduction. This should be underpinned by the results of funded research and/or consensus regarding a safe and efficient vital sign monitoring schedule and appropriate maximum permitted response times to clinical deterioration.

\section{CONCLUSIONS}

The 'deteriorating patient' policies of the hospitals studied varied in their contents and often omitted precise instructions for staff. We recommend that individual hospitals review these documents, and that research and/or consensus are used to develop a national algorithm regarding the response to patient deterioration. 


\section{ACKNOWLEDGEMENTS}

We would like to acknowledge the invaluable assistance of the Director of Nursing, NHS Improvement (NHSI), Dr. Ruth May, who contacted the Directors of Nursing/Chief Nurses in 152 acute NHS England (NHSE) Hospitals on our behalf. We would also like to thank the Directors of Nursing/Chief Nurses who returned the 'deteriorating patient' policies/guidelines in use in their hospitals.

\section{CURRENT AFFILIATIONS OF AUTHORS}

Mr. Simon Freathy is now Senior Clinical Quality Officer, NHS South Eastern Hampshire CCG, Hampshire, UK.

Professor Lisette Schoonhoven is now Professor of Nursing Science, UMC Utrecht, Utrecht, The Netherlands.

Professor Greta Westwood is now Chief Operating Officer, Florence Nightingale Foundation, London, UK.

\section{FUNDING}

The research was funded by the National Institute for Health Research (NIHR), Collaboration for Leadership in Applied Health Research and Care (NIHR CLAHRC) Wessex and Portsmouth Hospitals NHS Trust. The views expressed are those of the authors and not necessarily those of the NHS, the NIHR or the Department of Health and Social Care. 


\section{REFERENCES}

1. Royal College of Physicians. National Early Warning Score (NEWS) - Standardising the assessment of acute-illness severity in the NHS. Report of a working party. London; 2012.

2. Royal College of Physicians. National Early Warning Score (NEWS) 2 Standardising the assessment of acute-illness severity in the NHS. 2017.

3. National Institute for Health and Clinical Excellence: Acute ill patients in hospital: recognition of and response to acute illness in adults in hospital. NICE clinical guideline No. 50. London; 2007.

4. Smith GB, Osgood VM, Crane S. ALERT - a multiprofessional training course in the care of the acutely ill adult patient. Resuscitation. 2002;52:281-6.

5. Resuscitation Guidelines. The ABCDE approach. https://www.resus.org.uk/resuscitationguidelines/abcde-approach/ (accessed 15 ${ }^{\text {th }}$ March 2019)

6. Haig KM, Sutton S, Whittington J. SBAR: A Shared Mental Model for Improving Communication Between Clinicians. Jt Comm J Qual Patient Saf. 2006;32:167-75.

7. Smith GB, Prytherch DR, Schmidt P, et al. Hospital-wide physiological surveillance - A new approach to the early identification and management of the sick patient. Resuscitation. 2006;71:19-28.

8. Featherstone P, Chalmers T, Smith GB. RSVP: a system for communication of deterioration in hospital patients. Br J Nurs. 2008;17:860-4.

9. Beckett DJ, Gordon CF, Paterson R, et al. Improvement in out-of-hours outcomes following the implementation of Hospital at Night. QJM. 2009;102:539-46.

10. National Institute for Health and Care Excellence. Patient Group Directives. Medicines practice guideline (MPG2), London 2013.

11. Cheema K. NEWS baseline survey acute results. Patient Safety Measurement Unit; 2018. Available from: http://psmu.improvement.nhs.uk/workstreams/deterioration/news-baseline-survey (accessed 02/12/2018).

12. Smith GB. In-hospital cardiac arrest: is it time for an in-hospital 'chain of prevention'? Resuscitation. 2010;81:1209-11.

13. Clarke SP. Failure to rescue: lessons from missed opportunities in care. Nursing Inquiry. 2004;11:67-71. 
14. Barwise A, Thongprayoon C, Gajic O, Jensen J, Herasevich V, Pickering BW. Delayed Rapid Response Team Activation Is Associated With Increased Hospital Mortality, Morbidity, and Length of Stay in a Tertiary Care Institution. Crit Care Med. 2016;44:54-63.

15. Sankey CB, McAvay G, Siner JM, Barsky CL, Chaudhry SI. "Deterioration to Door Time": An Exploratory Analysis of Delays in Escalation of Care for Hospitalized Patients. J Gen Intern Med. 2016;31:895-900.

16. Harris S, Singer M, Sanderson C, Grieve R, Harrison D, Rowan K. Impact on mortality of prompt admission to critical care for deteriorating ward patients: an instrumental variable analysis using critical care bed strain. Intensive Care Med. 2018;44:606-615.

17. Smith GB, Recio-Saucedo A, Griffiths $P$. The measurement frequency and completeness of vital signs in general hospital wards: An evidence free zone? Int J Nurs Stud. 2017 Sep;74:A1-A4. doi: 10.1016/j.jinurstu.2017.07.001. Epub 2017 Jul 4.

18. Hands C, Reid E, Meredith P, et al. Patterns in the recording of vital signs and early warning scores: compliance with a clinical escalation protocol. BMJ Qual Saf. 2013;22:719-26.

19. Griffiths P, Ball J, Maruotti A, et al., on behalf of the Missed Care Study Group. Nurse staffing, nursing assistants and hospital mortality: retrospective longitudinal cohort study. BMJ Qual Saf. 2018 http://dx.doi.org/10.1136/ bmjqs-2018-008043.

20. Adelstein BA, Piza MA, Nayyar V, Mudaliar Y, Klineberg PL, Rubin G. Rapid response systems: a prospective study of response times. J Crit Care. 2011;26:635.e11-635.e18.

21. Leotsakos A, Zheng H, Croteau R, et al. Standardization in patient safety: the WHO High 5s project. Int J Qual Health Care. 2014;26:109-16.

22. Rozich JD, Howard RJ, Justeson JM, Macken PD, Lindsay ME, Resar RK. Standardization as a Mechanism to Improve Safety in Health Care. Jt Comm J Qual Saf. 2004;30:5-14.

23. Shearer B, Marshall S, Buist MD, et al. What stops hospital clinical staff from following protocols? An analysis of the incidence and factors behind the failure of bedside clinical staff to activate the rapid response system in a multi-campus Australian metropolitan healthcare service. BMJ Qual Saf. 2012;21:569-75.

24. Wakeam E, Hyder JA, Ashley SW, Weissman JS. Barriers and Strategies for Effective Patient Rescue: A Qualitative Study of Outliers. Jt Comm J Qual Patient Saf. 2014;40:503-513. 
25. McGregor C, Chohan S, O'Reilly J. Collaborative approach to reducing cardiac arrests in an acute medical unit. BMJ Open Quality 2017;6:e000026. doi:10.1136/bmjoq-2017-000026.

26. Morris A, Owen HM, Jones K, Hartin J, Welch J, Subbe CP. Objective patient-related outcomes of rapid-response systems - a pilot study to demonstrate feasibility in two hospitals. Crit Care Resusc. 2013;15:33-39. 


\section{Legends for Figures}

Figure 1:

NEWS thresholds and triggers. Reproduced from: Royal College of Physicians. National Early

Warning Score (NEWS): Standardising the assessment of acute illness severity in the NHS. Report of a working party. London: RCP, 2012.

Figure 2:

Clinical response to the NEWS trigger thresholds. Reproduced from: Royal College of Physicians.

National Early Warning Score (NEWS): Standardising the assessment of acute illness severity in the NHS. Report of a working party. London: RCP, 2012.

Figure 3:

Early warning scoring system in use in 55 hospitals.

Figure 4:

Recommended actions following medium-risk and high-risk response failure 
Table 1: Minimum frequency of observation for different early warning score (EWS) risk categories

\begin{tabular}{|c|c|c|c|c|c|c|}
\hline \multirow{2}{*}{$\begin{array}{l}\text { Minimum } \\
\text { observation } \\
\text { frequency }\end{array}$} & \multicolumn{3}{|c|}{ NEWS } & \multicolumn{3}{|c|}{ EWS other than NEWS } \\
\hline & $\begin{array}{c}\text { Low-risk } \\
\text { (NEWS>0) } \\
(n=36)\end{array}$ & $\begin{array}{c}\text { Medium } \\
\text {-risk } \\
(n=36)\end{array}$ & $\begin{array}{l}\text { High- } \\
\text { risk } \\
(n=36)\end{array}$ & $\begin{array}{c}\text { Low-risk } \\
(E W S>0) \\
(n=19)\end{array}$ & $\begin{array}{c}\text { Medium } \\
\text {-risk } \\
(n=19)\end{array}$ & $\begin{array}{c}\text { High-risk } \\
(\mathrm{n}=19)\end{array}$ \\
\hline 12-hourly & & & & $\begin{array}{c}1 \\
(5.3 \%)\end{array}$ & & \\
\hline 6-12 hourly & $4(11.1 \%)$ & & & & & \\
\hline 4-12 hourly & & & & $\begin{array}{c}4 \\
(21.1 \%)\end{array}$ & & \\
\hline 4-8 hourly & $\begin{array}{c}1 \\
(2.8 \%)\end{array}$ & & & & & \\
\hline 6-hourly & $\begin{array}{c}6 \\
(16.7 \%)\end{array}$ & & & & & \\
\hline 4-6 hourly & $\begin{array}{c}15 \\
(41.7 \%)\end{array}$ & & & $\begin{array}{c}1 \\
(5.3 \%) \\
\end{array}$ & & \\
\hline 4-hourly & $\begin{array}{c}9 \\
(25 \%)\end{array}$ & & & $\begin{array}{c}8 \\
(42.1 \%)\end{array}$ & & \\
\hline 2-4 hourly & & $\begin{array}{c}2 \\
(5.6 \%)\end{array}$ & & & & \\
\hline 1-4 hourly & & $\begin{array}{c}2 \\
(5.6 \%)\end{array}$ & & & $\begin{array}{c}3 \\
(15.8 \%)\end{array}$ & \\
\hline 2-hourly & & $\begin{array}{c}1 \\
(2.8 \%)\end{array}$ & & & $\begin{array}{c}3 \\
(15.8 \%)\end{array}$ & \\
\hline $\begin{array}{l}30 \text { minutes to } 2- \\
\text { hourly }\end{array}$ & & $\begin{array}{c}1 \\
(2.8 \%)\end{array}$ & & & & \\
\hline Hourly & & $\begin{array}{c}28 \\
(77.8 \%) \\
\end{array}$ & $\begin{array}{c}6 \\
(16.7 \%) \\
\end{array}$ & $\begin{array}{c}1 \\
(5.3 \%) \\
\end{array}$ & $\begin{array}{c}9 \\
(47.4 \%) \\
\end{array}$ & $\begin{array}{c}7 \\
(36.8 \%) \\
\end{array}$ \\
\hline 30 minutes - hourly & & & $\begin{array}{c}2 \\
(5.6 \%) \\
\end{array}$ & & & \\
\hline 30 minutes & & $\begin{array}{c}2 \\
(5.6 \%)\end{array}$ & $\begin{array}{c}4 \\
(11.1 \%)\end{array}$ & & $\begin{array}{c}4 \\
(21.2 \%)\end{array}$ & $\begin{array}{c}7 \\
(36.8 \%)\end{array}$ \\
\hline 15 minutes & & & $\begin{array}{c}3 \\
(8.3 \%)\end{array}$ & & & $\begin{array}{c}2 \\
(10.5 \%)\end{array}$ \\
\hline Hourly - continuous & & & $\begin{array}{c}1 \\
(2.8 \%)\end{array}$ & & & \\
\hline Continuous & & & $\begin{array}{c}20 \\
(55.6 \%)\end{array}$ & & & $\begin{array}{c}3 \\
(15.8 \%)\end{array}$ \\
\hline Nurse to decide & $\begin{array}{c}1 \\
(2.8 \%)\end{array}$ & & & $\begin{array}{c}3 \\
(15.8 \%)\end{array}$ & & \\
\hline $\begin{array}{l}\text { Minimum observation } \\
\text { frequency not stated }\end{array}$ & & & & $\begin{array}{c}1 \\
(5.3 \%)\end{array}$ & & \\
\hline
\end{tabular}


Table 2: Maximum response times for different early warning score (EWS) risk categories

\begin{tabular}{|l|r|r|r|r|}
\hline \multirow{2}{*}{ Maximum response time } & \multicolumn{2}{c|}{ NEWS } & \multicolumn{2}{c|}{$\begin{array}{c}\text { EWS other than NEWS } \\
\text { (n= 19) }\end{array}$} \\
\cline { 2 - 5 } & $\begin{array}{c}\text { Medium-risk } \\
(\mathbf{n = 3 6 )}\end{array}$ & $\begin{array}{c}\text { High-risk } \\
(\mathbf{n = 3 6 )}\end{array}$ & $\begin{array}{c}\text { Medium-risk } \\
(\mathbf{n}=\mathbf{1 9})\end{array}$ & $\begin{array}{c}\text { High-risk } \\
(\mathbf{n}=\mathbf{1 9})\end{array}$ \\
\hline 2 hours & & & $1(5.3 \%)$ & \\
\hline 1 hour & $6(16.7 \%)$ & & $4(21.1 \%)$ & $1(5.3 \%)$ \\
\hline 30 minutes & $17(47.2 \%)$ & $7(19.4 \%)$ & $10(52.6 \%)$ & $7(36.8 \%)$ \\
\hline 20 minutes & $1(2.8 \%)$ & $1(2.8 \%)$ & & \\
\hline 15 minutes & $1(2.8 \%)$ & $6(16.7 \%)$ & & $2(10.5 \%)$ \\
\hline 10 minutes & & $2(5.6 \%)$ & & $5(26.3 \%)$ \\
\hline 'Immediate' & $2(5.6 \%)$ & $15(41.7 \%)$ & & $1(5.3 \%)$ \\
\hline 'Urgent' & $5(13.9 \%)$ & & & \\
\hline 'Emergency' & & $2(5.6 \%)$ & & $3(15.8 \%)$ \\
\hline Call cardiac arrest team & & $1(2.8 \%)$ & & \\
\hline Maximum response time not stated & $4(11.1 \%)$ & $2(5.6 \%)$ & $4(21.1 \%)$ & \\
\hline
\end{tabular}

EWS $=$ Early Warning Score; NEWS = National Early Warning Score 


\section{Figure 1:}

NEWS thresholds and triggers. Reproduced from: Royal College of Physicians. National Early Warning Score (NEWS): Standardising the assessment of acute illness severity in the NHS. Report of a working party. London: RCP, 2012.

\begin{tabular}{|c|c|}
\hline NEW scores & Clinical risk \\
\hline 0 & \multirow{2}{*}{ Low } \\
\hline Aggregate 1-4 & \\
\hline $\begin{array}{c}\text { RED score } \\
\text { (Individual parameter scoring 3) }\end{array}$ & Medium \\
\hline Aggregate 5-6 & High \\
\hline Aggregate 7 \\
or more
\end{tabular}

\section{The NEWS trigger system aligned to the scale of clinical risk}

${ }^{*}$ RED score refers to an extreme variation in a single physiological parameter (ie a score of 3 on the NEWS chart, coloured RED to aid identification and represents an extreme variation in a single physiological parameter). The consensus of the NEWS Development and Implementation Group (NEWSDIG) was that extreme values in one physiological parameter (eg heart rate $\leq 40$ beats per minute, or a respiratory rate of $\leq 8$ per minute or a temperature of $\leq 35^{\circ} \mathrm{C}$ ) could not be ignored and on its own required urgent clinical evaluation. 
Figure 2:

Clinical response to the NEWS trigger thresholds. Reproduced from: Royal College of Physicians. National Early Warning Score (NEWS): Standardising the assessment of acute illness severity in the NHS. Report of a working party. London: RCP, 2012.

\begin{tabular}{|c|c|c|}
\hline NEWS SCORE & $\begin{array}{c}\text { FREQUENCY OF } \\
\text { MONITORING }\end{array}$ & CLINICAL RESPONSE \\
\hline 0 & Minimum 12 hourly & $\begin{array}{l}\text { - Continue routine NEWS monitoring with } \\
\text { every set of observations }\end{array}$ \\
\hline $\begin{array}{c}\text { Total: } \\
1-4\end{array}$ & Minimum 4-6 hourly & $\begin{array}{l}\text { - Inform registered nurse who must assess } \\
\text { the patient; } \\
\text { - Registered nurse to decide if increased } \\
\text { frequency of monitoring and / or } \\
\text { escalation of clinical care is required; }\end{array}$ \\
\hline $\begin{array}{l}\text { Total: } \\
5 \text { or more } \\
\text { or } \\
3 \text { in one } \\
\text { parameter }\end{array}$ & $\begin{array}{l}\text { Increased frequency } \\
\text { to a minimum } \\
\text { of } 1 \text { hourly }\end{array}$ & $\begin{array}{l}\text { Registered nurse to urgently inform } \\
\text { the medical team caring for the patient; } \\
\text { - Urgent assessment by a clinician } \\
\text { with core competencies to assess acutely } \\
\text { ill patients; } \\
\text { - Clinical care in an environment with } \\
\text { monitoring facilities; }\end{array}$ \\
\hline $\begin{array}{c}\text { Total: } \\
7 \\
\text { or more }\end{array}$ & $\begin{array}{c}\text { Continuous monitoring of } \\
\text { vital signs }\end{array}$ & $\begin{array}{l}\text { Registered nurse to immediately inform } \\
\text { the medical team caring for the patient - } \\
\text { this should be at least at Specialist } \\
\text { Registrar level; } \\
\text { - Emergency assessment by a clinical } \\
\text { team with critical care competencies, } \\
\text { which also includes a practitioner/s with } \\
\text { advanced airway skills; } \\
\text { - Consider transfer of Clinical care to a } \\
\text { level } 2 \text { or } 3 \text { care facility, i.e. higher } \\
\text { dependency or ITU; }\end{array}$ \\
\hline
\end{tabular}


Figure 3:

Early warning scoring system in use in 55 hospitals

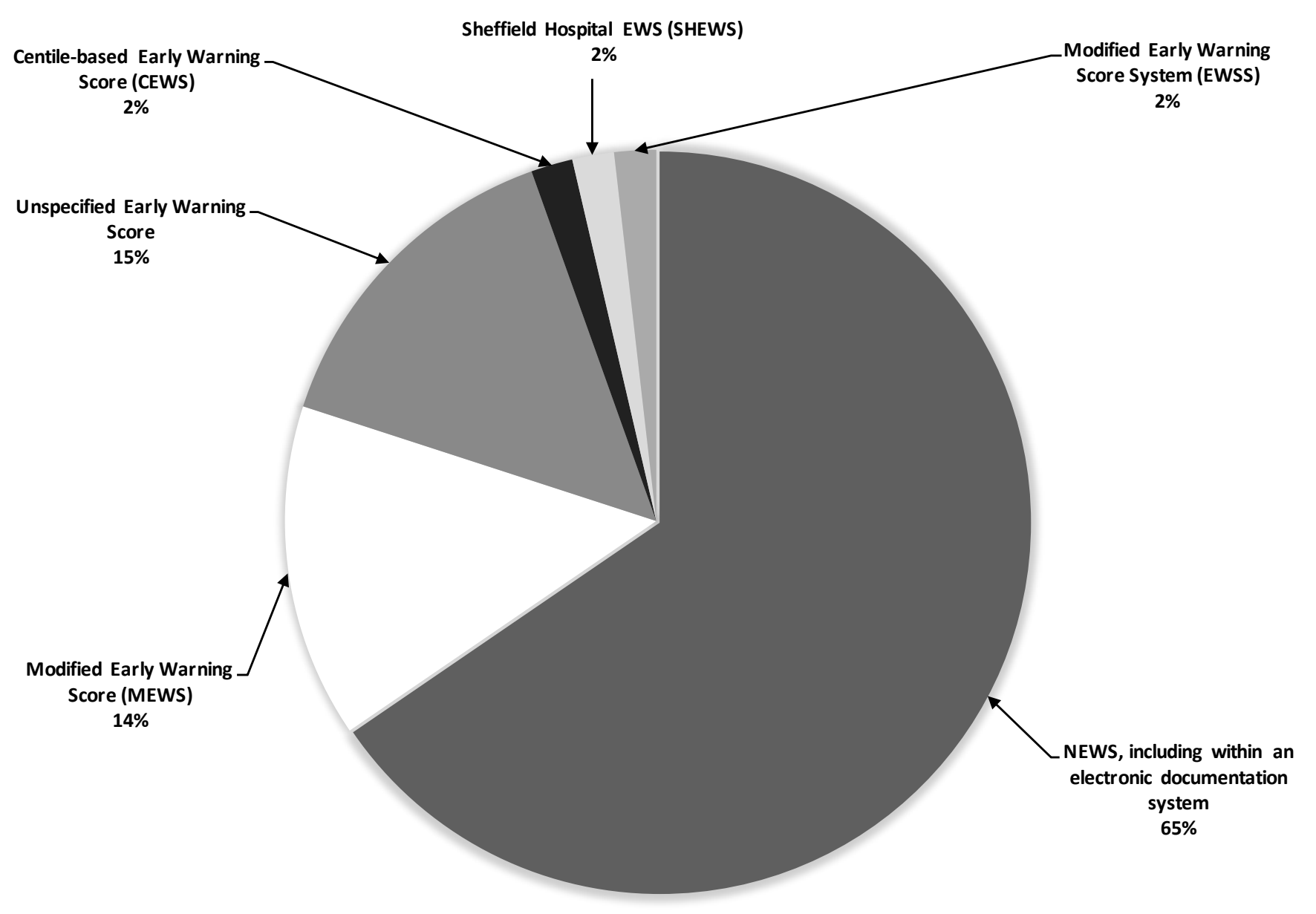


Figure 4:

Recommended actions following medium-risk and high-risk response failure

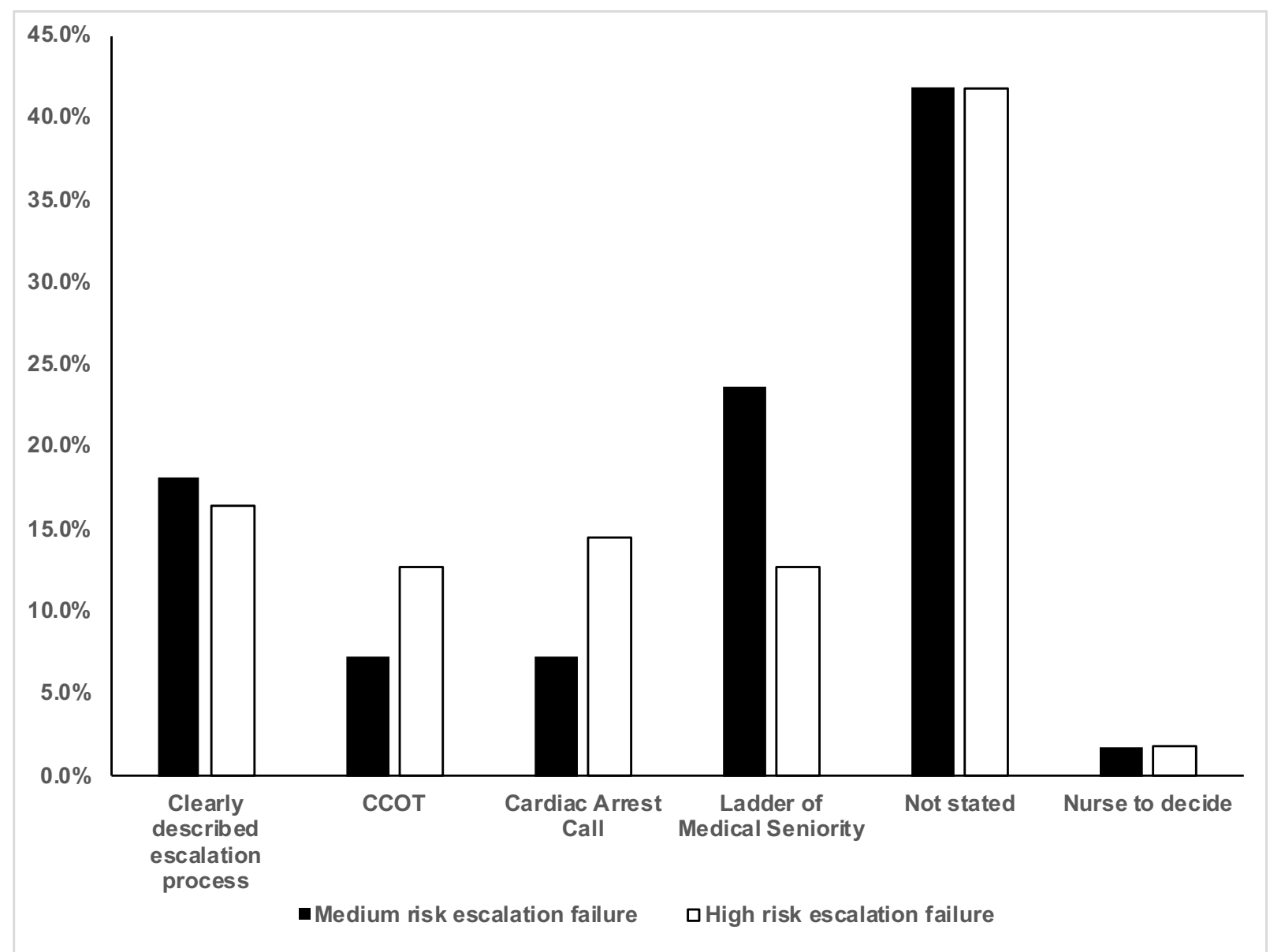

$\mathrm{CCOT}=$ Critical Care Outreach Team. 